quintile of SES (30\%) and fewer in the most deprived (10\%). There was a decreasing trend in dornase $\alpha$ treatment with increasing deprivation (least deprived 48\%: 43\%: 41\%: 41\%: most deprived $42 \%, \mathrm{p}=0.004)$ although when stratified by disease severity this was only significant among patients with mild disease $\left(70 \% \leq \mathrm{FEV}_{1} \%\right.$ predicted $<90 \%$ ). There were no differences in chronic $\mathrm{Pa}$ infections or $\mathrm{FEV}_{1} \%$ predicted.

Conclusion In the last 20 years, the proportion of adults attending specialist clinics has increased and the majority live near their clinics. Despite these improvements, there exist disparities in treatment by distance and SES and chronic Pa infections by model of care.

\section{P228 PATIENT EXPERIENCE OF A NURSE LED THERAPEUTIC PLEURAL ASPIRATION SERVICE}

doi:10.1136/thx.2010.151068.29

N Surange, J A Murray, S C 0 Taggart. Salford Royal NHS Foundation Trust, Salford, UK

Traditional models of care utilise the skills of a doctor to perform Therapeutic Pleural Aspiration (TPA). The procedure is often unplanned, rushed and performed by training doctors requiring supervision. At Salford Royal NHS Foundation Trust, we have trained the UKs first Lung Cancer Advanced Practitioner Nurse (LCAPN) to carry out TPA, as a bridge to alleviating chest symptoms prior to initiation of more definitive anti-cancer treatment and/or pleurodesis or as part of Best Suportive Care. From April 2009 to July 2010, our LCAPN carried out 41 planned TPAs independently in 23 individual patients with cancer related pleural effusion (11 lung cancers, 6 mesotheliomas, 6 other primaries) on the day ward, producing a total of $53327 \mathrm{ml}$ of fluid (mean $1300 \mathrm{ml}$ ). Patients were identified as suitable for TPA by the Lead Lung Cancer Clinician who used results from CT scan or same day Thoracic ultrasound scan to guide optimal site for needle placement using local anaesthesia (LA). Fluid was removed using the TRU-CLOSE suction drainage system. At the end of each procedure, patients were asked to complete a self-administered questionnaire based on their experience of the procedure, process of consent and comfort. Overall, the service was rated as excellent by $100 \%$ of patients. In particular, the service was rated highly for scheduling of TPA, information giving, consent, comfort and ability of LCAPN to perform the procedure. $76 \%$ of patients experienced either no pain or only mild discomfort and $21 \%$ experienced moderate discomfort, although this generally occurred at the end of the procedure.

Conclusion Nurse led TPA for cancer related pleural effusion is an acceptable model of care for alleviating symptoms prior to more definitive anti-cancer treatment or as part of Best Supportive Care.

\section{P229 CHOOSE AND BOOK: NOT A PATIENT-CENTRED SERVICE?}

doi:10.1136/thx.2010.151068.30

${ }^{1} \mathrm{~L}$ O'Byrne, ${ }^{2} \mathrm{~N}$ Roberts, ${ }^{1} \mathrm{M}$ R Partridge. ${ }^{1}$ Imperial College London, NHLI at Charing Cross Hospital, London, UK; ${ }^{2}$ Department of Public Health and Health Policy, University of Glasgow, London, UK

Introduction As part of another study we monitored Choose and Book $(\mathrm{C} \& \mathrm{~B})$ referrals attending our respiratory clinic and asked their views on their referral process. High non-attendance and limited patient choice with the $\mathrm{C} \& \mathrm{~B}$ system has previously been observed elsewhere, despite it being intended as a patient-centred service enhancement. We sought to gain insight into non-attendance of $\mathrm{C} \& \mathrm{~B}$ referrals at our clinic by analysing the levels of satisfaction with the referral process expressed by those who attended.
Method C\&B non-attendance/cancellation rates were calculated for the initial study recruitment period. Patients attending clinic were asked to confirm their referral route and their satisfaction with the referral system via a nurse-administered questionnaire.

Results $47 / 57$ (82.5\%) C\&B patients attended clinic during the study recruitment period. $2 / 57(3.5 \%)$ rearranged to a different clinic. 8/57 (14\%) failed to attend or cancelled. 44/47 (93.6\%) patients who attended clinic responded to the questionnaire. 18/44 (40.9\%) patients reported limited or no choice regarding time/date or hospital location of the appointment. 4/18 (22.2\%) said the appointment was arranged by their GP. A further $7 / 18$ (38.9\%) seemed unaware of the C\&B system or that they had a choice. $5 / 18$ (27.8\%) would have chosen a different hospital and 3/18 (16.7\%) would have chosen a different date/time. $3 / 18$ (16.7\%) failed to get their preference using the online/telephone booking systems. In 12/ $44(27.3 \%)$ cases the GP either made or assisted with the booking: 4/ 12 (33.3\%) patients were happy for the GP to choose, 4/12 (33.3\%) described a consultative process, $2 / 12(16.7 \%)$ felt choice was limited and 2/12 (16.7\%) made no additional comment. Only 16/44 (36.4\%) mentioned using the telephone/online booking systems. 5/ $16(31.3 \%)$ commented that the choice of dates or location that this provided was important to them. Patient reported problems with the systems included limited options and inflexibility when booking or rearranging appointments and a lack of information. 12/44 $(27.3 \%)$ specifically mentioned that they liked the choice and convenience the system offered.

Conclusion Our findings suggest that a high proportion of respiratory patients do not exercise true choice with Choose and Book. This supports observations in other patient groups. System and process obstacles seem to be exacerbated by lack of patient awareness and may be contributing to high non-attendance rates.

\section{P230 INAPPROPRIATE REFERRALS TO THE RAPID ACCESS LUNG CLINIC (RALC)}

doi:10.1136/thx.2010.151068.31

S Huq, M Gautam, M Haris, A Ashish, M Ledson, M Walshaw. Liverpool Heart and Chest Hospital NHS Foundation Trust, Liverpool, UK

Introduction and Aims Urgent referral suspected lung cancer cases depends upon nationally agreed protocols (a suspicious chest x-ray, persistent haemoptysis in smokers over age 40, stridor and SVC obstruction). In order to best use resource intensive RALC facilities, it is important that these protocols are followed. We wished to look at inappropriate referrals to the RALC which serves our large lung cancer unit (450 cases per year).

Methods We looked at source of and reasons for referral, eventual placement of the referral, and the ultimate diagnosis of all inappropriate referrals during the calendar year 2009

Results Of 452 referrals, 97 (21\%) did not follow the protocol [68 (70\%) primary, 14 (14\%) secondary care, and 15 (15\%) from the A\&E department]; including 76 (78\%) with a 'suspicious chest $\mathrm{x}$ ray' and $6(6 \%)$ with 'haemoptysis'. In 46 , the chest x-ray report did not suggest cancer, 6 had a normal chest $x$-ray, 2 from primary care had no radiology, 9 from secondary care had CT scans not suggestive of lung cancer, 5 did not meet the haemoptysis referral criteria, 8 were under specialist care for lung cancer/other malignancies, 11 were under chest physician/surgeon review and 3 were inpatients. One preferred investigation elsewhere, 1 had already been processed through the RALC, and one was a nursing home resident. In every case, a lung cancer unit clinician communicated with the referrer and channelled these referrals in timely fashion to appropriate services: $51(53 \%)$ to a general chest clinic, $16(16 \%)$ to other hospital specialists, $15(15 \%)$ back to their GP, and one to palliative care. Ultimately, only $3(3 \%)$ were subsequently diagnosed with 